\title{
Enhancement of Intratumoral Chemotherapy with Cisplatin with or without Microwave Ablation and Lipiodol. Future Concept for Local Treatment in Lung Cancer
}

\author{
Wolfgang Hohenforst-Schmidt ${ }^{1}$, Paul Zarogoulidis ${ }^{2 凶}{ }^{\circledR}$, Joshua Stopek ${ }^{3}$, Efstratios Kosmidis ${ }^{4}$, Thomas Vogl ${ }^{5}$, \\ Bernd Linsmeier ${ }^{6}$, Kosmas Tsakiridis ${ }^{7}$, Sofia Lampaki², George Lazaridis ${ }^{8}$, Andreas Mpakas ${ }^{7}$, Robert \\ Browning 9 , Antonis Papaiwannou ${ }^{2}$, Antonis Drevelegas ${ }^{10}$, Sofia Baka11, Vasilis Karavasilis ${ }^{8}$, Ioannis \\ Mpoukovinas ${ }^{12}$, J Francis Turner ${ }^{13}$, Konstantinos Zarogoulidis², Johannes Brachmann ${ }^{1}$ \\ 1. II Medical Clinic, "Coburg” Hospital, University of Wuerzburg, Coburg, Germany \\ 2. Pulmonary Department-Oncology Unit, "G. Papanikolaou” General Hospital, Aristotle University of Thessaloniki, Thessaloniki, Greece \\ 3. Covidien, U.S.A \\ 4. Veterinary School, Aristotle University of Thessaloniki, Greece \\ 5. Department of Diagnostic and Interventional Radiology, Goethe University of Frankfurt, Frankfurt, Germany \\ 6. Department of Thoracic Surgery, Medinos Clinic Sonneberg, Sonnerberg, Germany \\ 7. Department of Thoracic Surgery,"Saint Luke" Private Hospital, Panorama, Thessaloniki, Greece \\ 8. Oncology Department, "Papageorgiou" General Hospital, Aristotle University of Thessaloniki, Thessaloniki, Greece \\ 9. Pulmonary \& Critical Care Medicine, Interventional Pulmonology, National Naval Medical Center, Walter Reed Army Medical Center, Bethesda, U.S.A \\ 10. Radiology Department, "Interbalkan European Medical Center", Thessaloniki. Greece \\ 11. Oncology Department, "Interbalkan European Medical Center", Thessaloniki. Greece \\ 12. Oncology Department, "BioMedicine" Clinic, Thessaloniki, Greece \\ 13. Division of Interventional Pulmonology \& 2 Medical Oncology, Cancer Treatment Centers of America, Western Regional Medical Center, Goodyear, AZ
}

$\square$ Corresponding author: Paul Zarogoulidis, M.D, Ph. D, Pulmonary Department-Oncology Unit, “G. Papanikolaou” General Hospital, Aristotle University of Thessaloniki, Thessaloniki, Greece. Fax: 00302310992424; Mobile: 00306977271974; E-mail: pzarog@hotmail.com

(C) 2015 Ivyspring International Publisher. Reproduction is permitted for personal, noncommercial use, provided that the article is in whole, unmodified, and properly cited. See http://ivyspring.com/terms for terms and conditions.

Received: 2014.11.02; Accepted: 2014.12.13; Published: 2015.01.16

\begin{abstract}
Novel therapies for lung cancer are being explored nowadays with local therapies being the tip of the arrow. Intratumoral chemotherapy administration and local microwave ablation have been investigated in several studies. It has been previously proposed that lipiodol has the ability to modify the microenvironment matrix. In our current study we investigated this theory in BALBC mice. In total 160 BALBC mice were divided in eight groups: a) control, b) cisplatin, c) microwave, d) microwave and lipiodol, e) cisplatin and lipiodol, f) microwave and cisplatin, g) lipiodol and h) lipiodol, cisplatin and microwave. Lewis lung carcinoma cell lines $\left(10^{6}\right)$ were injected into the right back leg of each mouse. After the 8 th day, when the tumor volume was about $100 \mathrm{~mm}^{3}$ the therapy application was initiated, once per week for four weeks. Magnetic resonance imaging was performed for each tumor when a mouse died or when sacrificed if they were still alive by the end of the experiment (8-Canal multifunctional spool; NORAS MRI products, Gmbh, Germany). Imaging and survival revealed efficient tumor apoptosis for the groups b,c,d,e and f. However; severe toxicity was observed in group $h$ and no follow up was available for this group after the second week of therapy administration. Lipiodol in its current form does assist in a more efficient way the distribution of cisplatin, as the microwave apoptotic effect. Future modification of lipiodol might provide a more efficient method of therapy enhancement. Combination of drug and microwave ablation is possible and has an efficient apoptotic effect.
\end{abstract}

Key words: lung cancer, intratumoral, lipiodol, cisplatin, microwave ablation 


\section{Introduction}

Currently lung cancer treatment is based either on non-specific cytotoxic agents or targeted therapies.[1-5] Several molecular pathways are being investigated in order to identify novel targeted agents. [6] Targeted treatments for lung cancer have provided not only a treatment for lung cancer patients, but also an alternative treatment for the elder patients where non-specific cytotoxic agents cannot be applied due to adverse effects.[1] At the same time novel routes of administrating current targeted and non-specific cytotoxic agents are being investigated.[7-12] Local administration has the advantage of delivering a minimum drug dosage to the target site with less adverse effects. $[9,11]$ However; safety concerns still remain to be elicited.[9] Previous studies with non-specific cytotoxic agents being delivered intratumorally in lung cancer patients have provided positive results.[7, 13] Pulmonary physicians have the equipment to deliver local treatment within a lesion either with the bronchoscope or the endobronchial ultrasound.[7, 14] However; the tumor consistency still remains an obstacle for efficient drug diffusion. The extracellular matrix, tumor density and drug formulation interact as outcome parameters. Different tumors have different density and there are currently two methods of drug transportation the active and the passive transportation.[15] Based on the tumor type drug design must be the first factor considered. Extracellular modification is a strategy that has to be considered in order to enhance the diffusion of a drug within the tumor.[16] [17] It has been previously suggested that liposomal compounds have the ability to alter the extracellular matrix and enhance the antitumoral activity of several agents by enhancing the oxidative and nitrative stress.[18] In the study by Solazzo et. al. [18] radiofrequency ablation was combined with liposomal cytotoxic agents and increased antitumor efficiency was observed. Radiofrequency (RF) ablation is used for several solid tumors including lung cancer.[19] The major limitation of this treatment application is tumor size. RF ablation is not efficient for tumors greater than $5 \mathrm{~cm}$ [20], therefore combination therapies have been investigated. Combination therapies have focused on chemotherapeutic agents and agents which increase electrical and heat conductivity.[21, 22] Another method of enhancing the RF efficiency was to apply a chemotherapeutic agent (liposomal doxorubicin) which prolonged the apoptosis results of RF locally in the tumor site.[18] Blank liposomes have been also applied in combination with RF ablation and the same efficiency with liposomal chemotherapeutic agents was observed.[22, 23] It is therefore hypothesized that indeed liposomal compounds have the ability to prolong locally the effect of
RF ablation. In our current study we aimed to investigate whether we could enhance the antitumor activity of RF ablation with or without cisplatin with the additional administration of lipiodol. Lipiodol is a poppyseed oil used by injection as a radio-opaque contrast agent that is used to outline structures in radiological investigations. It is used in chemoembolization applications as a contrast agent in follow-up imaging.[24, 25] There is also an important safety issue with lipiodol intravasation (leakage) of the fluid into the venous system which has caused complications in the past. However; this adverse effect can be used as an advantage for solid tumors enhancing the penetration and diffusion of locally administered drug within a tumor. In the study by Sato et. al. [25] Lipiodol was combined with granulocytemacrophage colony-stimulating factor (GM-CSF) in order to produce a prolonged local immunotherapeutic effect in hepatocellular carcinoma.

\section{Methods}

\section{Mice}

One hundred sixty BALBC mice age 7-8 weeks old were purchased from the experimental laboratory of "Theiageneio" Anticancer Hospital, and were divided in eight groups. The Institute has the following authorization for production and experimentation on mice EL 25 BIO 011 and EL 25 BIO 013. The mice included were isolated ( 1 per cage) in a temperature-controlled room on 12-hour light-dark cycle and were allowed free access to food and water. The Lewis lung carcinoma cell line was obtained by ATCC $\left(\mathrm{CRL}-1642^{\mathrm{TM}}\right)$. The cells were routinely cultured in $25-\mathrm{cm}^{2}$ tissue culture flasks containing RPMI (ATCC, 30-2002) supplemented with $10 \%$ fetal bovine serum (Biochrom) according to the supplier's instruction. The cell line was incubated at 37o C in 5\% CO2. The doubling time of the cell line was 21 hours.[26] At confluence, cells were harvested with $0.25 \%$ trypsin and then were re-suspended at $1,5 \times 10^{6}$ cells in $0.15 \mathrm{ml}$ PBS (Phosphate Buffered Saline, Dulbecco, Biochrom) which was used to inject the mice. The suspension was inoculated subcutaneously (27-guage needle, $1,5 \times 10^{6}$ cells) in the back of the mice. The tumor volume was measured once weekly using bidimensional diameters (caliper) with the equation $\mathrm{V}=1 / 2 a b^{2}$, where the $a$ represents the length and $b$ the width $\left(\mathrm{mm}^{3}\right)$. The tumor was grown on the back of the mice. The animals were randomly divided into eight groups of 20 , when the tumor volume of $\sim 100 \mathrm{~mm}^{3}$ was reached. a) control, b) cisplatin, c) microwave, d) microwave and lipiodol, e) cisplatin and lipiodol, f) microwave and cisplatin, g) lipiodol and h) lipiodol, cisplatin and microwave. 


\section{Chemotherapy agent and Lipiodol}

The non-specific cytotoxic agent cisplatin/hospira 100mg/100ml, ONCO-TAIN ${ }^{\mathrm{TM}}$, HOSPIRA UK, LIMITED was obtained from our pulmonary oncology department (Pulmonary Department-Oncology Unit, "G. Papanikolaou" General Hospital, Aristotle University of Thessaloniki, Thessaloniki, Greece). (Figure 1.) Lipiodol was kindly provided from the Department of Diagnostic and Interventional Radiology, Goethe University of Frankfurt, Frankfurt, Germany by Professor Thomas Vogl.

\section{Microwave Ablation System}

The system Valleylab ${ }^{\mathrm{TM}}$ MW Ablation Generator manufactured for Valleylab, a division of Tyco Healthcare Group LP Boulder, Boulder, CO, USA was kindly provided by Joshua Stopek. (Figure 2.)

\section{MRI}

Magnetic resonance imaging was performed to the tumor on mouse when a specimen was sacrificed or died. The tumor was surgically removed from the body and stored in formalin. At the end of the experiment an MRI was performed for every tumor specimen of each group. We did not perform MRIs in specimens immediately after a therapeutic administration; no animal was sacrificed after one drug/therapy application. (Figure 3.) In order for the MRIs to be properly performed we used an 8-Canal multifunctional coil kindly provided by Professor Thomas Vogl (NORAS MRI products, GmbH, Ger- many). All MRIs were performed in the Department of Diagnostic and Interventional Radiology, Goethe University of Frankfurt, Frankfurt, Germany under the personal supervision of Professor Thomas Vogl.

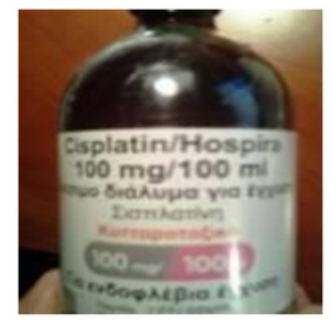

Figure 1. Non-specific cytotoxic agent cisplatin/hospira $100 \mathrm{mg} / 100 \mathrm{ml}$, ONCO-TAIN ${ }^{\mathrm{TM}}$, HOSPIRA UK, LIMITED

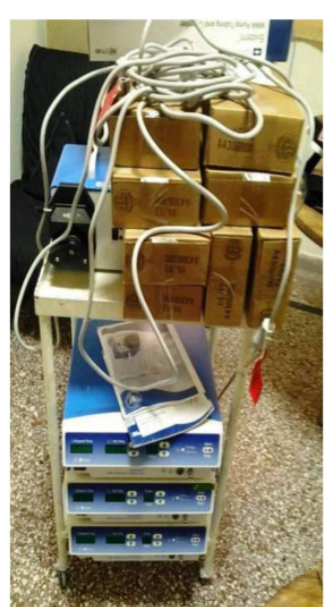

Figure 2. System Valleylab ${ }^{\text {TM }} M W$ Ablation Generator manufactured for Valleylab, a division of Tyco Healthcare Group LP Boulder, Boulder, CO, USA.

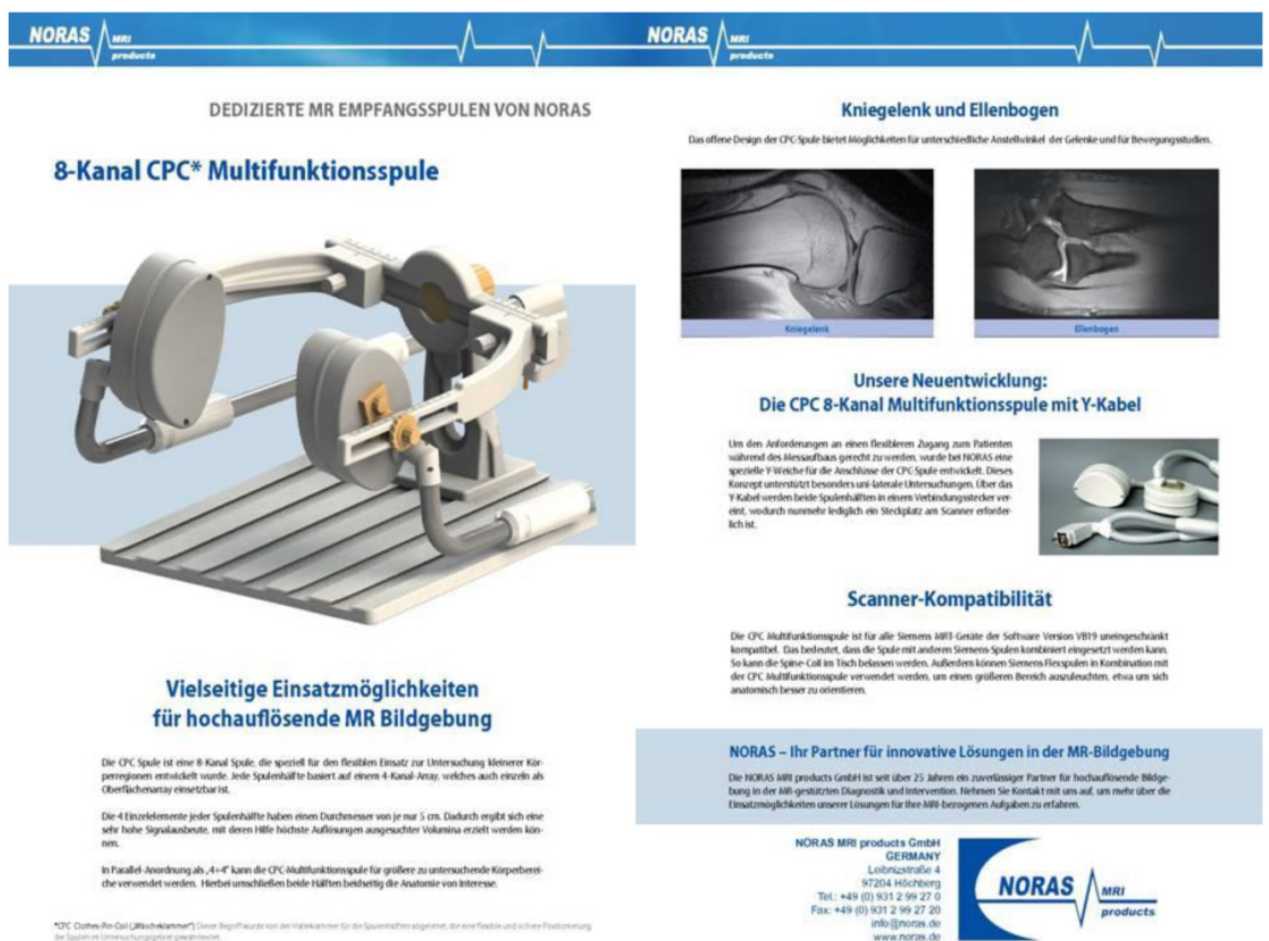

Figure 3. 8-Canal multifunctional coil (NORAS MRI products, GmbH, Germany) 


\section{Administration}

Cisplatin, and lipiodol were administered with an insulin syringe not in one spot, but in three different spots as indicated in Figure 4. The reason for this methodology was the concept of enhancing the therapy or lipiodol effect by distributing the drug in different parts of the tumor. On the other hand the microwave ablation spike was inserted in the center of the tumor, since the tumor was not larger than one $\mathrm{cm}$ in diameter. As it is indicated by the manufacturer (http://surgical.covidien.com/products/ablation-sys tems/microwave-ablation/evident-mwa-antennas) there is no need to insert an additional spike. The application was performed with 45 watts over 10 minutes. We used a $17 \mathrm{~cm}$ spike as this was available to us. Each spike was only $0.5-1 \mathrm{~cm}$ inserted in the center of the tumor. Cisplatin dosage was $1 \mathrm{ml}$ divided in three sites, and the same was applied for lipiodol.

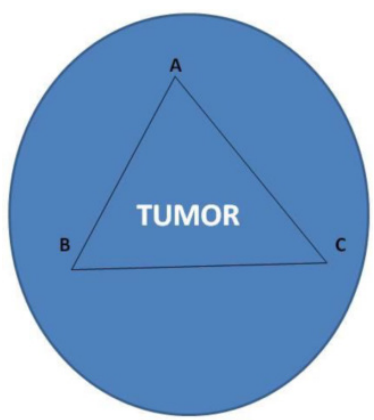

Figure 4. Letters $A, B$ and $C$ indicate the site of drug administration (Cisplatin and lipidilol) while in the center of the tumor the microwave ablation spike was inserted. We used a $17 \mathrm{~cm}$ spike as this was available to us and only $0.5-1 \mathrm{~cm}$ was inserted in the center of the tumor.

\section{Results}

Tumor necrosis and apoptosis were the major purposes of our study. Survival was also recorded. Our records and MRI findings indicate that a major toxicity was observed in the group where cisplatin, lipiodol and microwave were applied. None of the animals survived more than a week. Moreover; hemorrhage apart from necrosis was observed inside the tumors. Survival was the following for the groups: microwave (25days) $\geq$ cisplatin (22 days) $>$ control $(20$ days $)>$ lipiodol (16 days) $>$ microwave plus lipiodol (16 days) $>$ cisplatin plus lipiodol $(12)>$ cisplatin plus microwave $(12$ days $)>$ lipiodol plus cisplatin plus microwave (5 days). (Figure 5-12.)

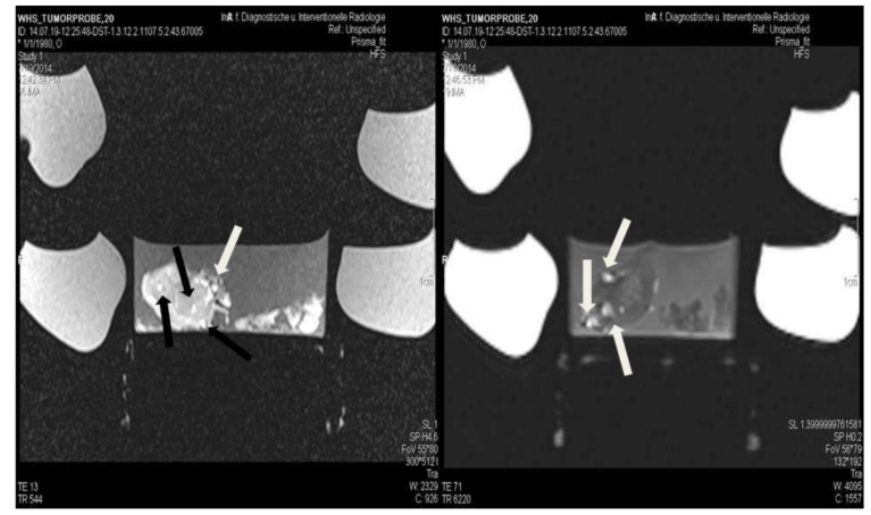

Figure 5. Lipiodol group; T1 sequence: Heterogeneous appearance in the central part of the tumor, low intensity indicates necrosis. T2 sequence: High signal intensity indicates necrosis. White and black arrows indicate lipiodol solution within the tumor. Necrosis is not extensive as presented in the current figures.

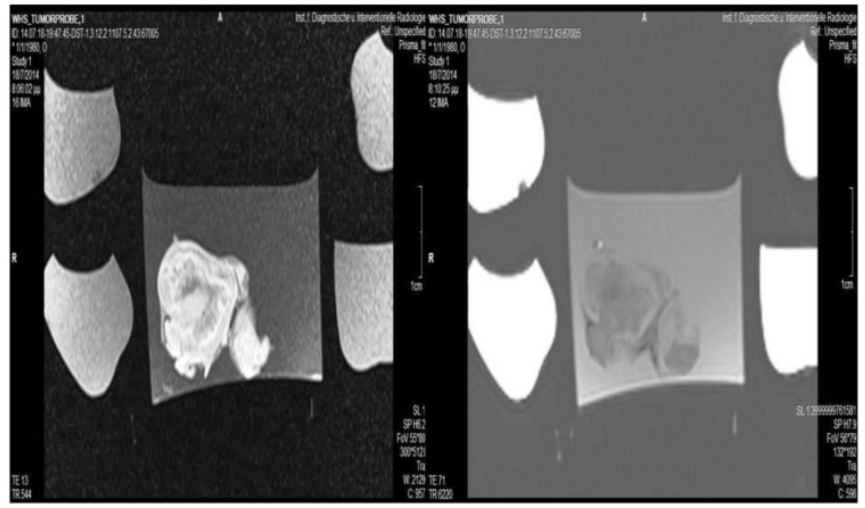

Figure 6. Control group; T1 sequence: Heterogeneous appearance in the central part of the tumor, low intensity indicates necrosis. T2 sequence: High signal intensity indicates necrosis. The necrosis observed in the center of the tumor is attributed to the necrosis that is normally observed within tumors.

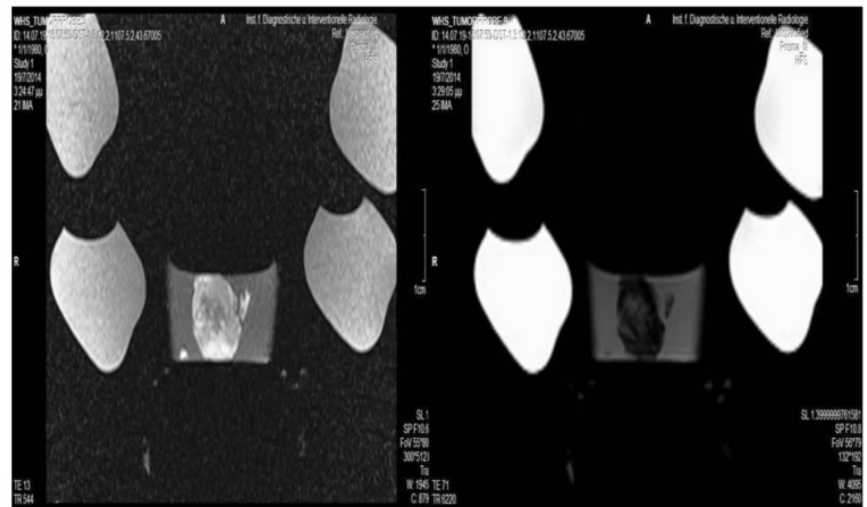

Figure 7. Cisplatin group; T1 sequence: Heterogeneous appearance in the central part of the tumor, low intensity indicates necrosis. T2 sequence: High signal intensity indicates necrosis. 


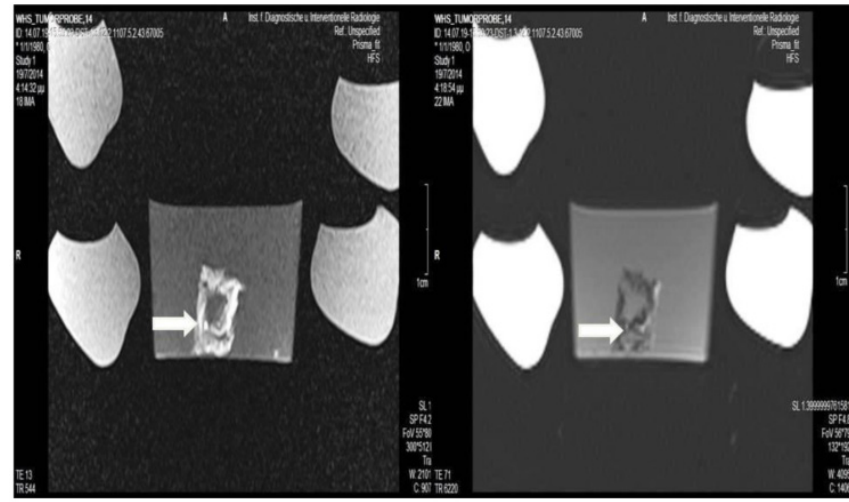

Figure 8. Lipidiolol and cisplatin; T1 sequence: Heterogeneous appearance in the central part of the tumor, low intensity indicates necrosis. T2 sequence: High signal intensity indicates necrosis. White formations are lipiodol molecules entrapped within the tumor tissue (white arrows).

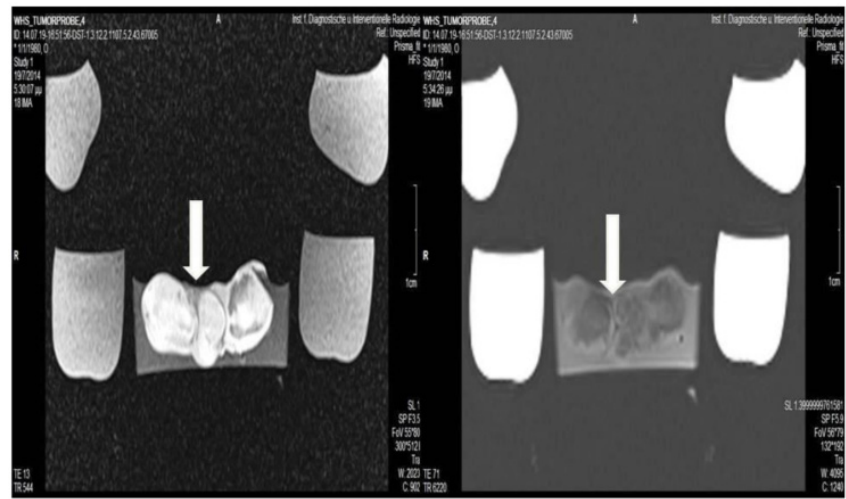

Figure 9. Microwave group; T1 sequence: Heterogeneous appearance with hypodensity sites indicating possible necrosis mostly in the periphery and in around the area where the spike was inserted. T2 sequence: Heterogeneous appearance with hyperdensity sites indicating possible necrosis mostly in the periphery and around the area where the spike was inserted. White arrow indicates area where the microwave spike was inserted.

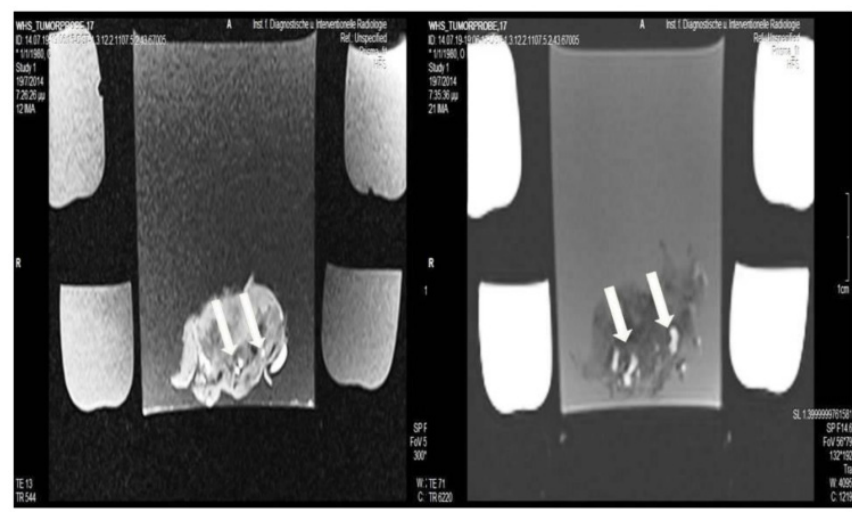

Figure 10. Lipiodol, cisplatin and microwave group; T1 sequence: Heterogeneous appearance with hypodensity sites indicating necrosis. T2 sequence: Heterogeneous appearance with hyperdensity sites indicating necrosis with hemorrhage around the spike wound. White formations are lipiodol molecules entrapped within the tumor tissue (white arrows).

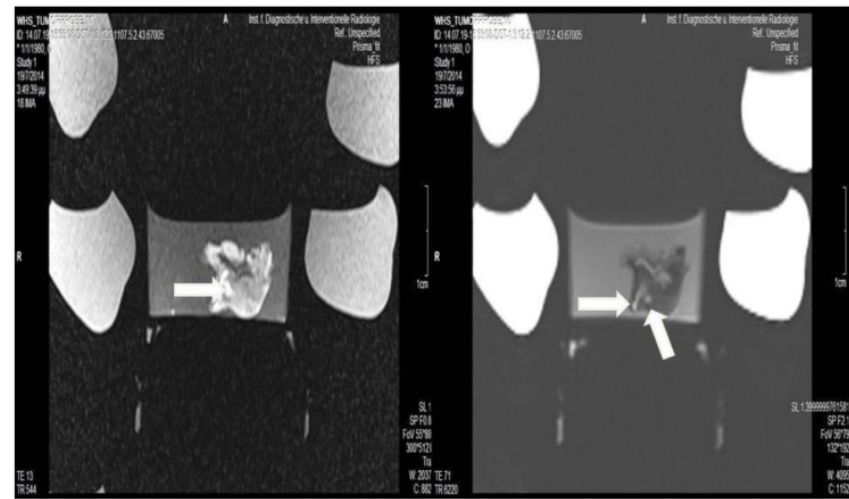

Figure 11. Lipiodol and microwave group; T1 sequence: Heterogeneous appearance with hypodensity sites indicating necrosis. T2 sequence: Heterogeneous appearance with hyperdensity sites indicating necrosis. White formations are lipiodol molecules entrapped within the tumor tissue.

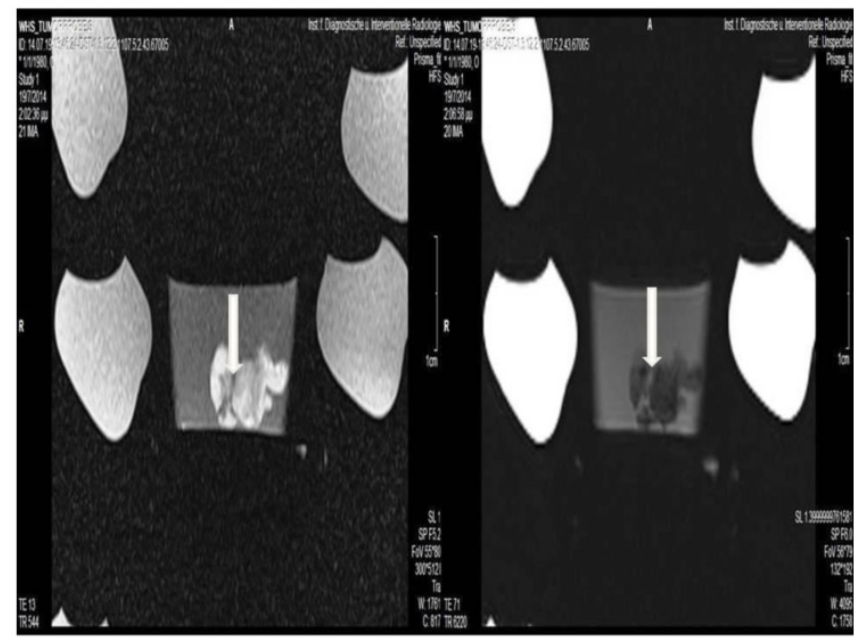

Figure 12. Cisplatin and Microwave; $T 1$ sequence: Heterogeneous appearance in the central part of the tumor, low intensity indicates necrosis. T2 sequence: High signal intensity indicates necrosis. White arrow indicates area where the microwave spike was inserted.

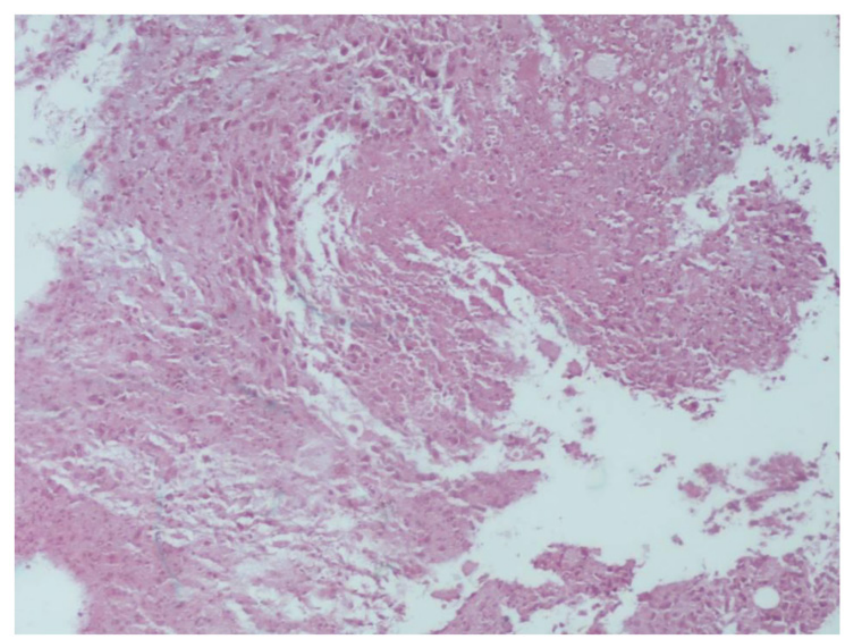

Figure 13. Cisplatin plus lipiodol group with $\mathrm{HE} \times 100$ 


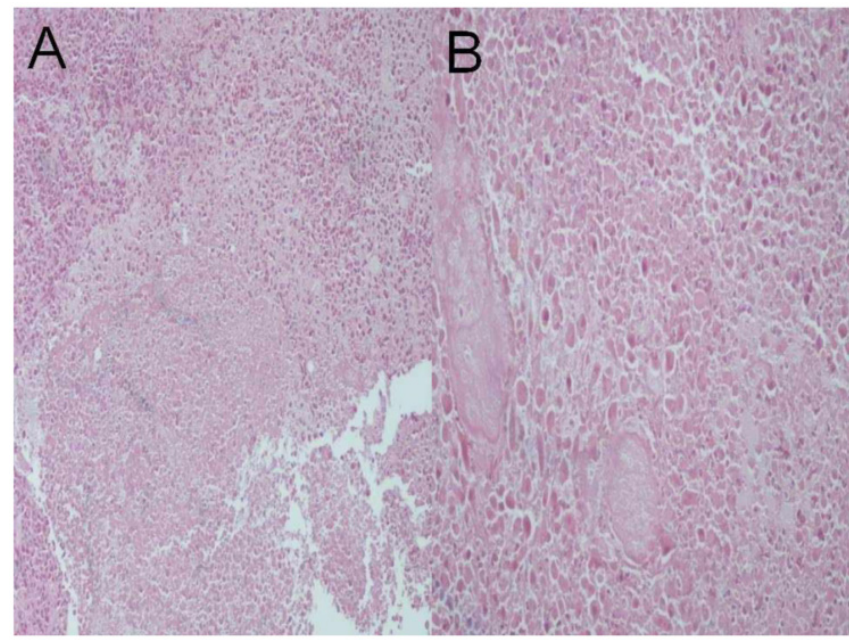

Figure 14. Cisplatin plus microwave group; a) HEX 100, b) HE X 200

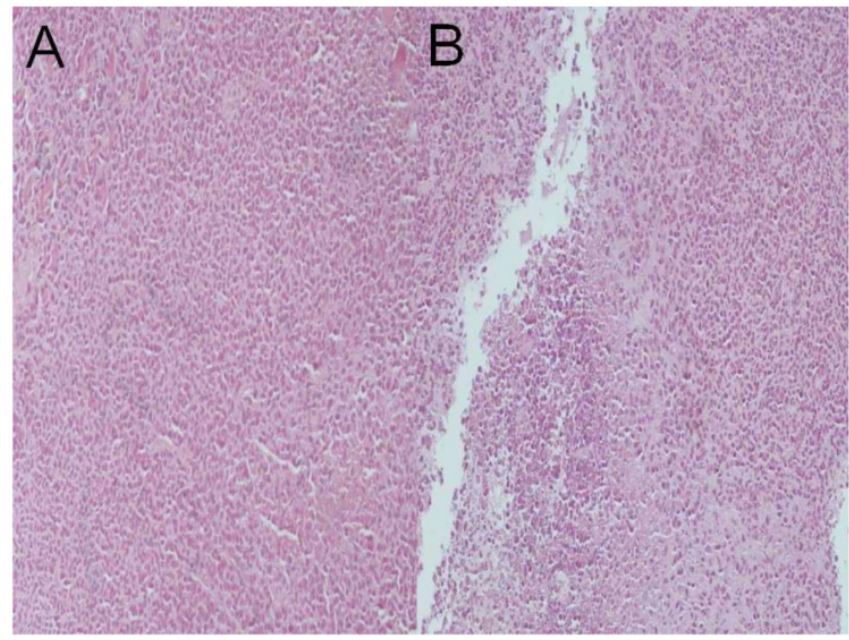

Figure 15. Lipiodol plus microwave group; a) HE x 100, b) HE x 100

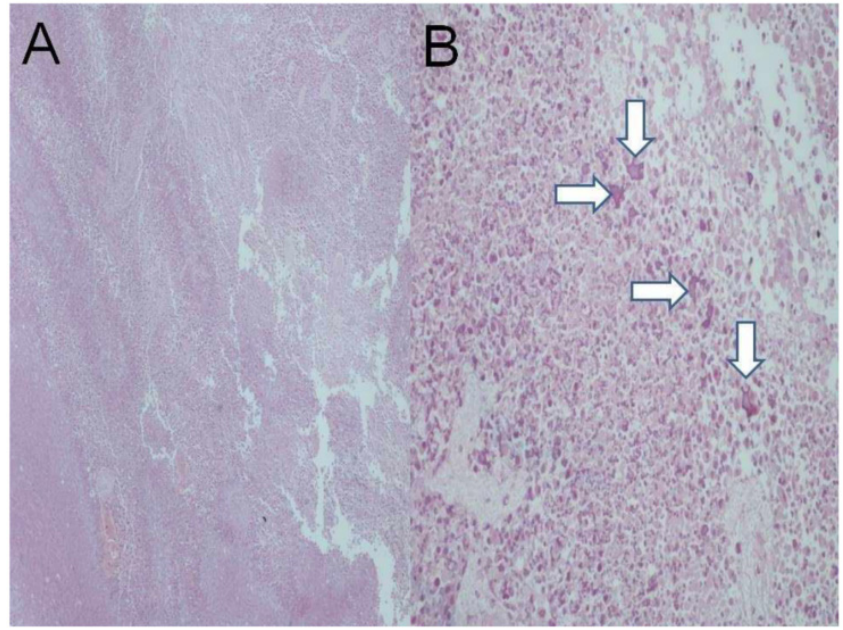

Figure 16. Lipiodol group; a) HE $\times 40$ (central necrosis is observed), b) $\mathrm{HE} \times$ 200 , white arrows indicate possibly lipiodol particles.

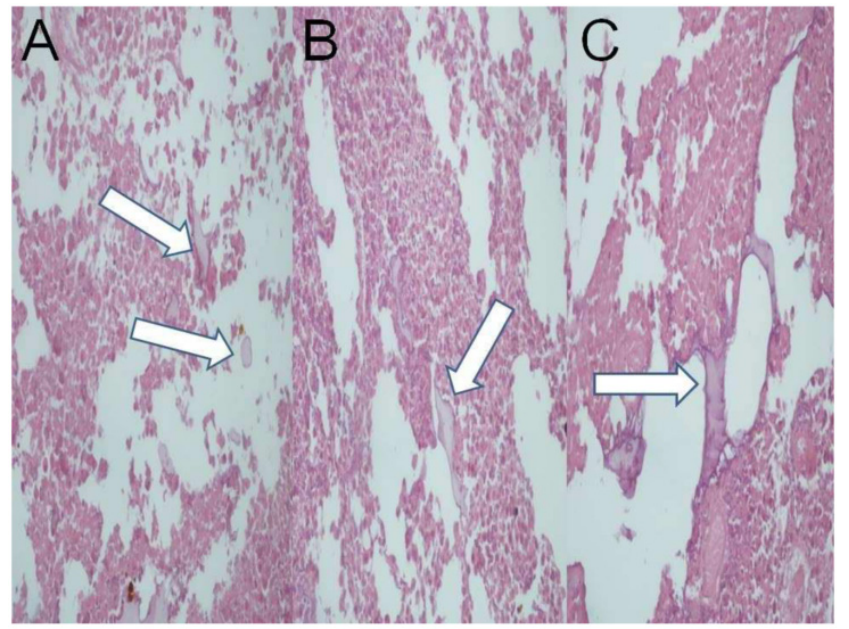

Figure 17. Lipiodol plus cisplatin plus microwave; a) HEX 200, b) HEX 200, c) HE X 200. (White arrows possibly indicate lipiodol)

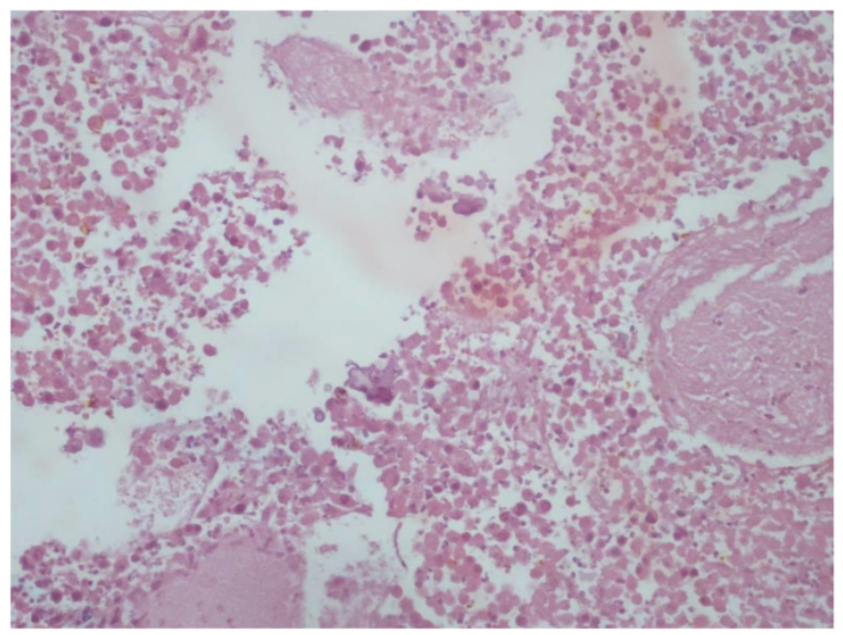

Figure 18. Microwave group; a) HE X 200, b) HE X 400

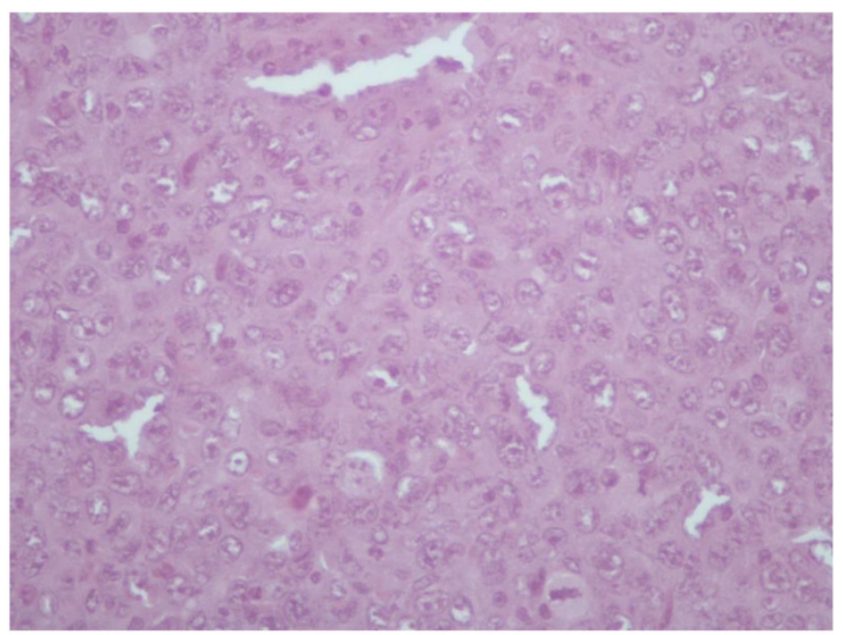

Figure 19. Control group; HE X 400 


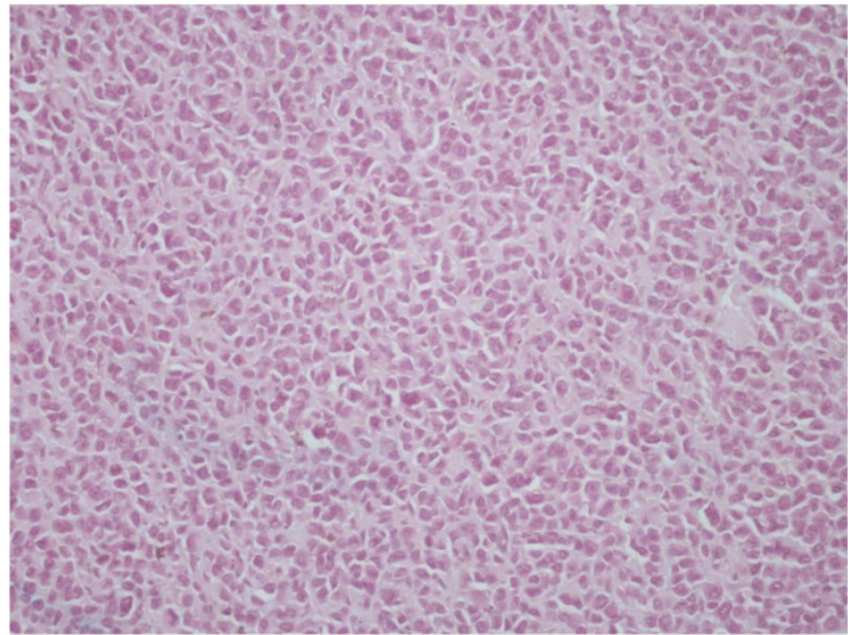

Figure 20. Cisplatin group; HE X 200. All pathology specimens were acquired 1 day after treatment administration (where applied).

\section{Discussion}

The application of microwave energy with spikes has proven to be an efficient method of local therapy for cancer and lung cancer in specific.[27-29] A recent study has presented data where this method is efficient and safer for the elderly $>75$ years old, or for patients not able to undergo chemotherapy.[27] The major drawback of this method is the short range of the thermal effect around the tip of each spike, therefore in many cases more than two spikes are inserted in the tumor with $1 \mathrm{~cm}$ distance from one and another in order to enhance the effect (http://surgical.covidien.com/products/ablation-sys tems/microwave-ablation/evident-mwa-antennas).

[29] Currently the guidance of the spike to the tumor lesion for lung cancer is possible with the assistance of Computed Tomography (CT) or Cone Beam CT, however; novel techniques are currently investigated with that of ultrasound being the newest.[30] Furthermore; a three dimensional magnetic guidance system is currently being developed.[31] Microwave ablation therapy has proven efficient for lesions $<3 \mathrm{~cm}$, distant to vessels and recurrence is rare in these situations.[32] It has been observed that when the microwave energy is applied for at least three minutes the tissue where the spike/s are inserted expands initially up to $25 \%$ and then the contraction takes place.[33] Moreover; in a recent study the novel treatment of cryoablation provided superior results when it was compared to radiofrequency ablation or microwave ablation, for hepatocellular carcinoma.[34] Possibly this methodology could be persued for lung cancer as well in the future. In another study by Sheng S. et. al. [35] microwave ablation was applied for hepatocellular carcinoma with the addition of recombinant adenovirus p53 gene (rAd-p53) and interleu- kin-2 intravenously. Complete remission of metastatic lesions to the lung was observed. The concept was to simultaneously stimulate the immune system and target the circulating cancer cells. However; this methodology is extremely costly. Microwave ablation is not without any adverse effects, in the case by Huang G. et. al. [36] invasive aspergillosis occurred after microwave ablation. Our current study was based on the concept that a lipophilic substance, such as lipiodol can enhance the thermal effect of microwave ablation. In the previous study by Solazzo SA et. al. [18] liposomal doxorubicin increased the effect of radiofrequency ablation by inducing tumor destruction by increasing cellular oxidative stress and accelerating apoptotic pathways. In the study by Vogl TJ. et. al. [37] it was observed that the thermal effect of laser-induced thermotherapy increases the activation of tumor-specific T lymphocytes. We therefore chose lipiodol as a lipid solution to investigate and observe whether this compound could enhance the chemotherapy effect of cisplatin or thermal effect of microwave ablation. Our results indicate the toxicity of $45 \mathrm{~W}$ and 10 minutes application for small tumors (group g; lipiodol, cisplatin and microwave), in a future study we will focus on developing an appropriate model for small animals and tumor volumes. It was observed that lipiodol is a very difficult solution to handle and as observed in the MRI figures 5-12, it is not distributed homogeneously. Based on this observation we conclude that it does not assist at all in the thermal effect of microwave ablation. Moreover; it does not assist in the homogeneous distribution of cisplatin, nor adds a sustained release effect to cisplatin absorption. We used the smallest doses possible for cisplatin and lipiodol, since the tumor model that we chose was very small. One of the major limitations of our study was that we did not have a dose escalation for cisplatin, lipiodol and microwave. Furthermore, it was observed that lipiodol, does not have an effect on its own. It has been proposed that nanoparticles could stimulate the dendritic cells (DC) and activate the immune system locally, because when nanoparticles/solutions of small molecules are inserted locally to the tumor they are recognized as foreign material and the T-cells are stimulated.[38] In conclusion; combination of cisplatin and microwave ablation could be applied as a method to enhance local therapy, however; more studies are needed to investigate proper dosage in comparison to tumor size. Additionally, we need a drug that can distribute homogeneously within the tumor after administration. Current chemotherapy drugs are not designed for this purpose. 


\section{Acknowledgments}

Drs Wolfgang Hohenforst-Schmidt and Paul Zarogoulidis would like to personally thank Professor Thomas Vogl and Joshua Stopek who provided the MRI spool, Lipiodol and Microwave ablation generator (ValleyLab ${ }^{\mathrm{TM}}$ ), without their motivation, help and useful insight this project would not have been fulfilled. The authors WHS, TV and PZ would like to thank Frank Huebner (Department of Diagnostic and Interventional Radiology, Goethe University of Frankfurt, Frankfurt, Germany) for his useful insights. PZ would also like to thank Alexandros Kalifatidis (Radiology Department, "Saint Luke" Private Hospital, Thessaloniki, Greece.) for his useful insights. PZ and EK would also like to thank Konstantino Tsirli (Laboratory of Physiology, Dept of Medicine, Aristotle University of Thessaloniki, University Campus, 54124, Thessaloniki, Greece. *Current address: Interdisciplinary Graduate Programme in the BRAIN and MIND Sciences, Faculty of Medicine, University of Crete, Greece.) for his assistance throughout the experiment.

\section{Conflict of Interest}

None to declare.

\section{References}

1. Domvri K, Zarogoulidis P, Darwiche K, Browning RF, Li Q, Turner JF, et al. Molecular Targeted Drugs and Biomarkers in NSCLC, the Evolving Role of Individualized Therapy. J Cancer. 2013; 4: 736-54. doi:10.7150/jca.7734.

2. Zarogoulidis K, Boutsikou E, Zarogoulidis P, Darwiche K, Freitag L, Porpodis $\mathrm{K}$, et al. The role of second-line chemotherapy in small cell lung cancer: a retrospective analysis. Onco Targets Ther. 2013; 6: 1493-500. doi:10.2147/OTT.S52330.

3. Kallianos A, Rapti A, Zarogoulidis P, Tsakiridis K, Mpakas A, Katsikogiannis $\mathrm{N}$, et al. Therapeutic procedure in small cell lung cancer. J Thorac Dis. 2013; 5: S420-4. doi:10.3978/j.issn.2072-1439.2013.09.16.

4. Zarogoulidis K, Zarogoulidis P, Darwiche K, Boutsikou E, Machairiotis N, Tsakiridis K, et al. Treatment of non-small cell lung cancer (NSCLC). J Thorac Dis. 2013; 5: S389-96. doi:10.3978/j.issn.2072-1439.2013.07.10.

5. Zaric B, Stojsic V, Tepavac A, Sarcev T, Zarogoulidis P, Darwiche K, et al. Adjuvant chemotherapy and radiotherapy in the treatment of non-small cell lung cancer (NSCLC). J Thorac Dis. 2013; 5: S371-7. doi:10.3978/j.issn.2072-1439.2013.05.16.

6. Cao Q, Zhao L, Wang P. [Advances in the Molecular Mechanisms and Prognostic Significance of EMT in Non-small Cell Lung Cancer]. Zhongguo Fei Ai Za Zhi. 2014; 17: 569-74. doi:10.3779/j.issn.1009-3419.2014.07.13.

7. Hohenforst-Schmidt W, Zarogoulidis P, Darwiche K, Vogl T, Goldberg EP, Huang $\mathrm{H}$, et al. Intratumoral chemotherapy for lung cancer: re-challenge current targeted therapies. Drug Des Devel Ther. 2013; 7: 571-83. doi:10.2147/DDDT.S46393.

8. Baliaka A, Zarogoulidis P, Domvri K, Hohenforst-Schmidt W, Sakkas A, Huang $\mathrm{H}$, et al. Intratumoral gene therapy versus intravenous gene therapy for distant metastasis control with 2-diethylaminoethyl-dextran methyl methacrylate copolymer non-viral vector-p53. Gene Ther. 2014; 21: 158-67. doi:10.1038/gt.2013.68.

9. Darwiche K, Zarogoulidis P, Karamanos NK, Domvri K, Chatzaki E, Constantinidis TC, et al. Efficacy versus safety concerns for aerosol chemotherapy in non-small-cell lung cancer: a future dilemma for micro-oncology. Future Oncol. 2013; 9: 505-25. doi:10.2217/fon.12.205.

10. Hohenforst-Schmidt W, Zarogoulidis P, Linsmeier B, Kioumis I, Li Q, Huang $\mathrm{H}$, et al. Enhancement of Aerosol Cisplatin Chemotherapy with Gene Therapy Expressing ABC10 protein in Respiratory System. J Cancer. 2014; 5: 344-50. doi:10.7150/jca.9021.

11. Zarogoulidis P, Eleftheriadou E, Sapardanis I, Zarogoulidou V, Lithoxopoulou $\mathrm{H}$, Kontakiotis T, et al. Feasibility and effectiveness of inhaled carboplatin in NSCLC patients. Invest New Drugs. 2012; 30: 1628-40. doi:10.1007/s10637-011-9714-5.
12. Zarogoulidis P, Petridis D, Ritzoulis C, Darwiche K, Spyratos D, Huang H, et al. Establishing the optimal nebulization system for paclitaxel, docetaxel, cisplatin, carboplatin and gemcitabine: back to drawing the residual cup. Int J Pharm. 2013; 453: 480-7. doi:10.1016/j.ijpharm.2013.06.011.

13. Celikoglu F, Celikoglu SI, Goldberg EP. Bronchoscopic intratumoral chemotherapy of lung cancer. Lung Cancer. 2008; 61: 1-12. doi:10.1016/j.lungcan.2008.03.009.

14. Celikoglu F, Celikoglu SI, York AM, Goldberg EP. Intratumoral administration of cisplatin through a bronchoscope followed by irradiation for treatment of inoperable non-small cell obstructive lung cancer. Lung Cancer. 2006; 51: 225-36. doi:10.1016/j.lungcan.2005.10.012.

15. Bae YH. Interview with Dr You Han Bae: ligand-mediated versus 'passive' targeting approaches in nanoparticle oncology research. Ther Deliv. 2012; 3: 933-6.

16. Elsaid KA, Ferreira L, Truong T, Liang A, Machan J, D'Souza GG. Pharmaceutical nanocarrier association with chondrocytes and cartilage explants: influence of surface modification and extracellular matrix depletion. Osteoarthritis Cartilage. 2013; 21: 377-84. doi:10.1016/j.joca.2012.11.011.

17. Grantab RH, Tannock IF. Penetration of anticancer drugs through tumour tissue as a function of cellular packing density and interstitial fluid pressure and its modification by bortezomib. BMC Cancer. 2012; 12: 214. doi:10.1186/1471-2407-12-214.

18. Solazzo SA, Ahmed M, Schor-Bardach R, Yang W, Girnun GD, Rahmanuddin $\mathrm{S}$, et al. Liposomal doxorubicin increases radiofrequency ablation-induced tumor destruction by increasing cellular oxidative and nitrative stress and accelerating apoptotic pathways. Radiology. 2010; 255: 62-74. doi:10.1148/radiol.09091196.

19. Dupuy DE, DiPetrillo $\mathrm{T}$, Gandhi $\mathrm{S}$, Ready $\mathrm{N}, \mathrm{Ng} \mathrm{T}$, Donat $\mathrm{W}$, et al. Radiofrequency ablation followed by conventional radiotherapy for medically inoperable stage I non-small cell lung cancer. Chest. 2006; 129: 738-45. doi:10.1378/chest.129.3.738.

20. Livraghi T, Goldberg SN, Lazzaroni S, Meloni F, Ierace T, Solbiati L, et al. Hepatocellular carcinoma: radio-frequency ablation of medium and large lesions. Radiology. 2000; 214: 761-8. doi:10.1148/radiology.214.3.r00mr02761.

21. Goldberg SN, Ahmed M, Gazelle GS, Kruskal JB, Huertas JC, Halpern EF, et al. Radio-frequency thermal ablation with $\mathrm{NaCl}$ solution injection: effect of electrical conductivity on tissue heating and coagulation-phantom and porcine $\begin{array}{llll}\text { liver study. 2001; } & \text { Radiology. 219: }\end{array}$ doi:10.1148/radiology.219.1.r01ap27157.

22. Ahmed M, Lukyanov AN, Torchilin V, Tournier H, Schneider AN, Goldberg SN. Combined radiofrequency ablation and adjuvant liposomal chemotherapy: effect of chemotherapeutic agent, nanoparticle size, and circulation time. J Vasc Interv Radiol. 2005; 16: 1365-71. doi:10.1097/01.RVI.0000175324.63304.25.

23. Goldberg SN, Girnan GD, Lukyanov AN, Ahmed M, Monsky WL, Gazelle GS, et al. Percutaneous tumor ablation: increased necrosis with combined radio-frequency ablation and intravenous liposomal doxorubicin in a rat breast tumor model. Radiology. 2002; 222: 797-804. doi:10.1148/radiol.2223010861.

24. Ahrar K, Gupta S. Hepatic artery embolization for hepatocellular carcinoma: technique, patient selection, and outcomes. Surg Oncol Clin N Am. 2003; 12: 105-26.

25. Sato T. Locoregional immuno(bio)therapy for liver metastases. Semin Oncol. 2002; 29: 160-7.

26. Bertram JS, Janik P. Establishment of a cloned line of Lewis Lung Carcinoma cells adapted to cell culture. Cancer Lett. 1980; 11: 63-73.

27. Acksteiner C, Steinke K. Percutaneous microwave ablation for early-stage non-small cell lung cancer (NSCLC) in the elderly: A promising outlook. J Med Imaging Radiat Oncol. 2014; doi:10.1111/1754-9485.12251.

28. Schneider T. [Thermal Ablation of Malignant Lung Tumours.]. Zentralbl Chir. 2014; doi:10.1055/s-0034-1368542.

29. Winokur RS, Du JY, Pua BB, Talenfeld AD, Sista AK, Schiffman MA, et al. Characterization of In Vivo Ablation Zones Following Percutaneous Microwave Ablation of the Liver with Two Commercially Available Devices: Are Manufacturer Published Reference Values Useful? J Vasc Interv Radiol. 2014. doi:10.1016/j.jvir.2014.08.014.

30. Wu W, Xue Y, Wang D, Xue J, Zhai W, Liang P. A simulator for percutaneous hepatic microwave thermal ablation under ultrasound guidance. Int $\mathrm{J}$ Hyperthermia. 2014;: 1-9. doi:10.3109/02656736.2014.957738.

31. Sindram D, Simo KA, Swan RZ, Razzaque S, Niemeyer DJ, Seshadri RM, et al. Laparoscopic microwave ablation of human liver tumours using a novel three-dimensional magnetic guidance system. HPB (Oxford). 2014. doi:10.1111/hpb.12315.

32. Leung U, Kuk D, D'Angelica MI, Kingham TP, Allen PJ, DeMatteo RP, et al. Long-term outcomes following microwave ablation for liver malignancies. $\mathrm{Br} \mathrm{J}$ Surg. 2014. doi:10.1002/bjs.9649.

33. Farina L, Weiss N, Nissenbaum $Y$, Cavagnaro M, Lopresto V, Pinto R, et al. Characterisation of tissue shrinkage during microwave thermal ablation. Int $\mathrm{J}$ Hyperthermia. 2014;: 1-10. doi:10.3109/02656736.2014.957250.

34. Ei S, Hibi T, Tanabe M, Itano O, Shinoda M, Kitago M, et al. Cryoablation Provides Superior Local Control of Primary Hepatocellular Carcinomas of $>2$ $\mathrm{cm}$ Compared with Radiofrequency Ablation and Microwave Coagulation Therapy: An Underestimated Tool in the Toolbox. Ann Surg Oncol. 2014; doi:10.1245/s10434-014-4114-7. 
35. Sheng S, Zheng J, Cui S, Cui X, Qian Z. Complete remission of multiple lung metastases after ablation of hepatocellular carcinoma by transarterial infusion with the p53 gene. Anticancer Drugs. 2014. doi:10.1097/CAD.0000000000000167.

36. Huang G, Liu Q, Ye X, Yang X, Wei Z, Li W, et al. Invasive pulmonary aspergillosis: A rare complication after microwave ablation. Int $\mathrm{J}$ Hyperthermia. 2014; 30: 412-7. doi:10.3109/02656736.2014.955064.

37. Vogl TJ, Wissniowski TT, Naguib NN, Hammerstingl RM, Mack MG, Munch $\mathrm{S}$, et al. Activation of tumor-specific $\mathrm{T}$ lymphocytes after laser-induced thermotherapy in patients with colorectal liver metastases. Cancer Immunol Immunother. 2009; 58: 1557-63. doi:10.1007/s00262-009-0663-1.

38. Seydoux E, Rothen-Rutishauser B, Nita IM, Balog S, Gazdhar A, Stumbles PA, et al. Size-dependent accumulation of particles in lysosomes modulates dendritic cell function through impaired antigen degradation. Int J Nanomedicine. 2014; 9: 3885-902. doi:10.2147/IJN.S64353. 